ARTICLE

\title{
Role of mutational reversions and fitness restoration in Zika virus spread to the Americas
}

\author{
Jianying Liu, ${ }^{1,6}$ Yang Liu ${ }^{2,6}$, Chao Shan², Bruno T. D. Nunes ${ }^{3}$, Ruimei Yun ${ }^{1}$, Sherry L. Haller ${ }^{1}$, Grace H. Rafael',

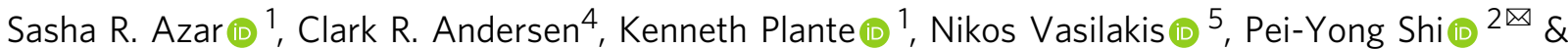 \\ Scott C. Weaver (iD ${ }^{1 凶}$
}

Zika virus (ZIKV) emerged from obscurity in 2013 to spread from Asia to the South Pacific and the Americas, where millions of people were infected, accompanied by severe disease including microcephaly following congenital infections. Phylogenetic studies have shown that ZIKV evolved in Africa and later spread to Asia, and that the Asian lineage is responsible for the recent epidemics in the South Pacific and Americas. However, the reasons for the sudden emergence of ZIKV remain enigmatic. Here we report evolutionary analyses that revealed four mutations, which occurred just before ZIKV introduction to the Americas, represent direct reversions of previous mutations that accompanied earlier spread from Africa to Asia and early circulation there. Our experimental infections of Aedes aegypti mosquitoes, human cells, and mice using ZIKV strains with and without these mutations demonstrate that the original mutations reduced fitness for urban, human-amplifed transmission, while the reversions restored fitness, increasing epidemic risk. These findings include characterization of three transmission-adaptive ZIKV mutations, and demonstration that these and one identified previously restored fitness for epidemic transmission soon before introduction into the Americas. The initial mutations may have followed founder effects and/or drift when the virus was introduced decades ago into Asia.

\footnotetext{
${ }^{1}$ World Reference Center for Emerging Viruses and Arboviruses, Institute for Human Infections and Immunity, and Department of Microbiology and Immunology, University of Texas Medical Branch, Galveston, TX 77555, USA. ${ }^{2}$ Department of Biochemistry and Molecular Biology and Institute for Human Infections and Immunity, University of Texas Medical Branch, Galveston, TX 77555, USA. ${ }^{3}$ Department of Arbovirology and Hemorrhagic Fevers, Evandro Chagas Institute, Ministry of Health, Ananindeua, Pará State, Brazil. ${ }^{4}$ Department of Preventive Medicine and Community Health, University of Texas Medical Branch, Galveston, TX 77555, USA. ${ }^{5}$ Department of Pathology, Center for Biodefense and Emerging Infectious Diseases, World Reference Center for Emerging Viruses and Arboviruses, and Institute for Human Infections and Immunity, University of Texas Medical Branch, Galveston, TX 77555, USA. ${ }^{6}$ These

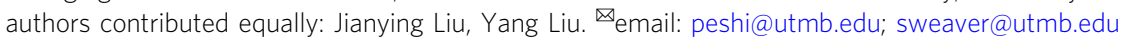


Z ika virus (ZIKV), an arthropod-borne virus (arbovirus) in the genus Flavivirus, discovered in $1947^{1}$, remained obscure with little association with human disease until 2007. Then, small outbreaks occurred in Gabon ${ }^{2}$ and Yap Island in Micronesia ${ }^{3}$, associated with mild dengue-like illness. In 2013, ZIKV spread to the South Pacific, where approximately half of the residents of French Polynesia were infected ${ }^{4}$, and an association with Guillain Barré syndrome (GBS) was discovered ${ }^{5}$. Spread to other areas of the South Pacific followed; then the first outbreak ever detected in the Americas was identified in 2015 northeastern Brazil ${ }^{6}$, accompanied by a dramatic increase in the incidence of microcephaly ${ }^{7}$ and other congenital malformations now termed congenital Zika syndrome $(\mathrm{CZS})^{8}$. ZIKV continued to spread to nearly all countries in the Americas with the continued association with GBS and CZS, leading the World Health Organization to declare in 2016 a Public Health Emergency of International Concern.

Early phylogenetic studies combined with ZIKV detections and seroprevalence indicated that the virus originated in and remains widespread in Africa, and was introduced many decades ago to Asia $^{9}$. However, the reason(s) for its dramatic spread to the Americas remain enigmatic. One hypothesis is that ZIKV increased its ability to be transmitted efficiently by mosquito vectors through adaptive mutations. This mechanism has been demonstrated for other arboviruses including West Nile ${ }^{10}$, Venezuelan equine encephalitis ${ }^{11}$, and chikungunya (CHIKV) viruses $^{12}$. RNA arboviruses exhibit high mutational frequencies due to their lack of proof-reading during genome replication, providing the opportunity for rapid adaptation to changing infection and transmission opportunities ${ }^{13}$. The first evidence supporting this adaptive evolution hypothesis for ZIKV came from studies of an A188V amino acid substitution in the nonstructural protein 1 (NS1-A188V) first detected in 2013, just before ZIKV spread to the South Pacific and the Americas. This substitution slightly enhances ZIKV transmission by Aedes (Stegomyia) aegypti mosquitoes ${ }^{14}$, the principal epidemic vector ${ }^{15}$. Another substitution, V473M in the envelope protein, increases viremia in nonhuman primates, also suggesting enhanced fitness for transmission ${ }^{16}$, and capsid substitution T106A, first detected in 2012, enhances murine neurovirulence ${ }^{16}$. Pre-membrane protein S17N enhances infectivity in human and mouse neural progenitor cells and causes more severe microcephaly in mice ${ }^{17}$, phenotypes not associated with more efficient transmission via viremia.

Here we report the results of further ZIKV phylogenetic analyses that reveal that four amino acid substitutions that preceded introductions into the South Pacific and the Americas represent direct reversions of previous mutations that occurred during or soon after ZIKV was introduced long ago into Asia from Africa. These reversions restored fitness declines for urban transmission that resulted from the initial mutations, increasing epidemic potential.

\section{Results}

Directly reverting Zika virus mutations. To further test the hypothesis that adaptive evolution enhanced epidemic ZIKV transmission, leading to dramatic spread, millions of infections, and the resulting detection of rare disease manifestations, we performed phylogenetic analyses to identify additional amino acid substitutions that preceded its spread to the Americas. Supplementary Fig. 1 shows a tree with four substitutions that preceded epidemic spread, including NS1-A188V ${ }^{14}$. The numbers of these substitutions among Asian strains are also shown. Tracings of these amino acids in trees are shown in Supplementary Fig. 2. Strikingly, these four substitutions all represented direct reversions of earlier mutations that accompanied spread from
Africa to Asia, or early circulation in Asia. None of these initial mutations was noted in earlier phylogenetic studies ${ }^{18}$.

The simplest explanation for this reversion pattern is that the pre-epidemic substitutions restored fitness (reproduction and survival ability) declines caused by founder effects and/or genetic drift during early, inefficient $A$. aegypti-borne interhuman transmission. This transmission inefficiency is consistent with the lack of recognized Zika outbreaks in Asia before 2010, and bottlenecks that accompany the arbovirus transmission cycle that can allow drift to dominate evolution if populations remain small. Founder effects result in a loss in genetic diversity that accompanies geographic introductions of small founder populations from a large, ancestral population. For human-amplified arboviruses like ZIKV, these typically involve a single infected traveler ${ }^{19}$. Furthermore, several virus population bottlenecks punctuate the arbovirus transmission cycle during mosquito infection and dissemination of virus to the salivary glands. The loss of genetic diversity can result in the fixation of random mutations, the majority of which are, by chance, deleterious for RNA viruses ${ }^{20}$ and other organisms. The hypothesis that African ZIKV strains lost fitness upon their introduction into Asia is also supported by the greater infectivity of African isolates for A. aegypti mosquitoes compared to Asian and American isolates $^{21-23}$, as well as their greater virulence in mouse models of human infection ${ }^{23,24}$.

As an initial test of the hypothesis that ZIKV underwent a fitness decline upon its introduction into Africa, followed by fitness restoration, we assessed the fitness of African, Asian, and American ZIKV strains. We used competition fitness assays, where ZIKV strains differing by as little as one amino acid were generated from cDNA clones and mixed in a roughly 1:1 ratio based on Vero cell plaque-forming units (PFU). This mixture was then used to initiate infections. Following appropriate incubation to allow the two strains to compete for replication and, in mosquitoes, dissemination to the salivary glands, the virus mixture was again quantified by Sanger sequencing of RT-PCR amplicons to estimate the ratios of mixed nucleotides using electropherogram peaks. The relative fitness value $\mathrm{w}$ was analyzed according to $\mathrm{w}=(\mathrm{f} 0 / \mathrm{i} 0)$, where $\mathrm{f0}$ is the final ratio of one competitor following infection, and $\mathrm{i} 0$ is the initial ratio in the inoculum or bloodmeal mixture; this ratio reflects the relative fitness advantage of one competitor over the other (Fig. 1a). This approach has major advantages over performing individual strain infections with numerous host replicates; each competition is internally controlled, eliminating host-to-host variation that can reduce the power of experiments, and the virus strain ratios can be assayed with more precision than individual virus titers. Thus, competition assays have been used for many studies of microbial fitness $^{25}$, including arboviruses ${ }^{26,27}$ and a coronavirus ${ }^{28}$.

Because the competition assay relies on electropherogram peak measurements from Sanger sequencing to quantify mutant ratios, with the potential for inconsistency, we validated the consistency and accuracy of this method. Supplementary Fig. 3 shows the great accuracy and consistency of this method. To confirm that our targeted 1:1 ratios were similar in mosquito experimental systems, where RNA:infectious virus ratios could differ based on different host-dependent levels of mutant infectivity, compared to those for Vero cells, we also determined infectious titers of the wt and mutants on C6/36 mosquito cells, and compared them with the Vero PFU ratios. These ratios were nearly indistinguishable with no significant differences (Supplementary Fig. 4), indicating that frequency-based differences in fitness based on potential mutation effects on initial infectivity were not a concern.

Fitness of major Zika virus lineages. Initially, we performed competition assays with wild-type (wt) ZIKV isolates representing the African, Asian pre-epidemic, and American epidemic 


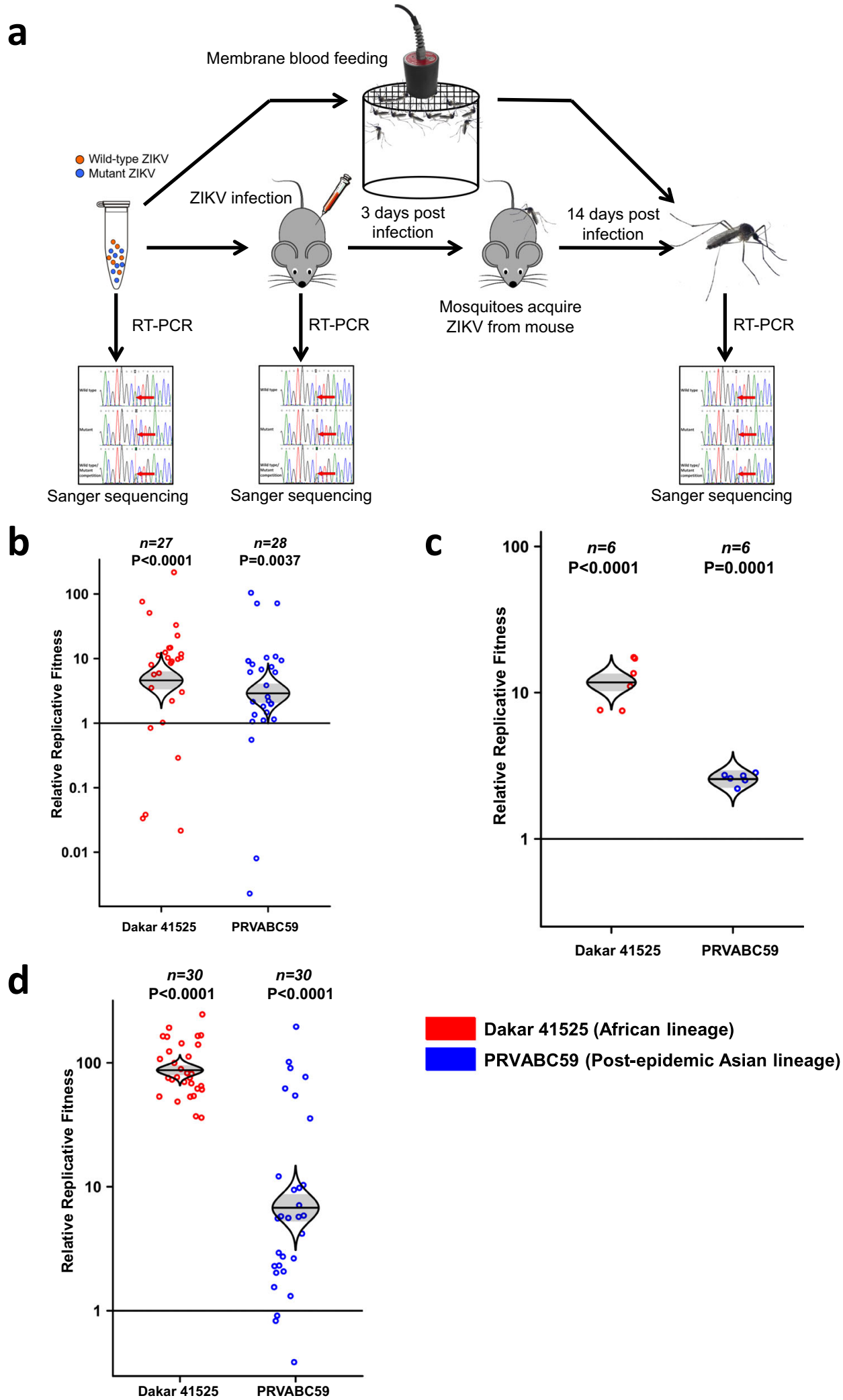

genotypes (Fig. 1). The highly susceptible Rockefeller strain of $A$. aegypti mosquitoes was used along with interferon type I receptor-deficient A129 mice, models for human infection ${ }^{29,30 .}$ Following infection with an approximately 1:1 mixture of the African Dakar 41525 and Asian Cambodian FSS13025 isolates, or an equivalent mixture of FSS130025 and the Puerto Rican RRVABC59 strain, samples from mosquito bodies and mice were evaluated for changes in the initial ratio, indications of competitive fitness (Fig. 1a). Following oral mosquito infection and incubation, the African isolate consistently and significantly won the competition with the Asian strain, and the Puerto Rican strain consistently outcompeted the Asian strain (Fig. 1b). These findings were reproducible when additional African, Asian, and American ZIKV isolates, as well as a Dominican Republic strain 


\begin{abstract}
Fig. 1 African lineage ZIKV and post-epidemic Asian lineage ZIKV had a fitness advantage versus pre-epidemic Asian lineage ZIKVs in both A129 mice and mosquitoes. a Experimental design of competition fitness assays. African lineage ZIKV and post-epidemic Asian lineage ZIKV were mixed with pre-epidemic Asian lineage ZIKV at an approximate ratio of 1:1 and these ratios were confirmed by RT-PCR, followed by Sanger sequencing of replicons and polymorphic nucleotide peaks analysis. The ZIKV mixture was fed to mosquitos through an artificial membrane or a A129 mouse at the viremia peak 3 days post-infection. Mosquitoes were sacrificed after 14 days of incubation, the ZIKV population amplified by RT-PCR, and the amplicon Sanger-sequenced. Mouse blood and organs were collected 3 or 8 days post-infection, respectively. All mosquito and mouse specimens were subjected to RT-PCR amplification and Sanger sequencing to compare the ZIKV strain ratio after competition, and each point represents a single mosquito or mouse sample. Relative fitness values were compared among multiple competition replicates to determine if fitness of the competitors differed. Figure adapted from Fig. 1a in Ref. ${ }^{14}$. b Fitness comparison between African (Dakar 41525), Asian pre-epidemic (FSS13025), and American (PRVABC59) ZIKV strains in A. aegypti. The relative replicative fitness value $w$ was analyzed according to $w=(f 0 / i 0)$, where i0 is the initial ratio of the Dakar 41525 or PRVABC59 competitor to the FSS13025 strain, determined from Sanger sequence electropherogram peaks, and f0 is the final ratio. c, Fitness comparison of African (Dakar 41525), Asian pre-epidemic (FSS13025), and American (PRVABC59) ZIKV strains in mouse viremia, using the same method as for $\mathbf{b}$. $\mathbf{d}$ Fitness comparison of African (Dakar 41525), Asian pre-epidemic (FSS13025), and American (PRVABC59) ZIKV strains in mosquitoes after feeding on the viremic mice shown in c, using the same method as for $\mathbf{b}$. b-d. The distribution of the model-adjusted means is illustrated by catseye plots with shaded \pm standard errors (SE) overlaid by scatterplots of individual relative fitness values shown on the log (base-10) scale such that comparisons are against a null value of 1. $N$, numbers represent biologically independent samples shown on the top of each figure. Source data are provided as a Source Data file. $\mathbf{b}$, d, The results were pooled from 3 independent repeats. c The results were collected from a single experiment.
\end{abstract}

of $A$. aegypti with a low level of colonization (F6) (Supplementary Fig. 5). After three days of competition following subcutaneous infection of A129 mice to mimic human infection, the same relative fitness results were obtained when viremic blood (the vertebrate host compartment where major selection for efficient mosquito infection occurs) was analyzed (Fig. 1c). Analysis of individual organ samples from mice on day 8 (after viremia had subsided to eliminate this confounding source of virus in organs) showed that these competition outcomes were consistent for replication throughout the mouse (Supplementary Fig. 6). Mosquitoes that fed upon these viremic mice were also evaluated after extrinsic incubation, with the same results of greater fitness of the African versus Asian ZIKV strain, and greater fitness of the American versus Asian strain (Fig. 1d).

Next, primary human cells believed to be involved in seeding viremia were used for competition assays: fibroblasts and keratinocytes $^{31,32}$. The mixed ZIKVs were inoculated into cells and the culture supernatant was harvested, RT-PCR-amplified and Sanger sequenced 3 days post-infection (Supplementary Fig. 7a). The results showed that the African isolate and postepidemic American strains always outcompeted the pre-epidemic Asian strain in both cell types (Supplementary Fig. 7b, c). In all of these experimental models, the changes in competitor ratios appeared to be greater between the African versus Asian strains compared to the Asian versus American strains. Overall, these data supported the hypothesis that ZIKV underwent a major loss of fitness upon its introduction into Asia, followed by a partial restoration of fitness upon introduction into the Americas. Microcephaly and other central nervous system involvement are rare and not known to play a major role in generating viremia, so there should not be the major selection for CNS tropism to enhance viremia and transmission by mosquitoes. Therefore, microcephaly may represent a chance pathogenic outcome that did not result from positive selection. Also, sexual ZIKV transmission is believed to play a minor role compared to vector-borne transmission.

Revised Hypothesis. Based on the evidence discussed above, we developed a revised adaptation hypothesis, depicted in Fig. 2a, that the 4 initial amino acid substitutions (and possibly others not undergoing reversion) represent founder effects that reduced the fitness of ZIKV for transmission in the epidemic cycle by reducing $A$. aegypti transmissibility and/or human viremia levels. The reversion of these 4 mutations, and possibly other adaptive mutations, then partially restored ZIKV fitness, allowing for efficient spread to the Americas and major epidemics.
Fitness effect of reversions. To test this hypothesis that the 4 revertant mutations restored ZIKV fitness, we used $A$. aegypti mosquitoes and A129 mice in more detailed studies of the combinations of four amino acid substitutions that underwent reversion. For mosquito infections, we assayed bodies to evaluate infection and initial replication, legs to sample the hemolymph, which contains virus that has disseminated from the digestive tract (midgut) into the hemocoel for access to the salivary glands, and saliva collected in vitro to assess the virus population available for transmission (Fig. 2b). Competition between the African ZIKV isolate and a mutant containing the four initial amino acid substitutions resulted in a consistent advantage for the African isolate in all mosquito samples (Fig. 2c), as did infection of A129 mice as sampled in major organs and blood (Fig. 2d, Supplementary Fig. 8a). In contrast, when the four reversion substitutions were placed into the pre-epidemic Asian strain to recapitulate ZIKV evolution just before introduction into the Americas, a major fitness gain occurred (Fig. 2e, f, Supplementary Fig. $8 \mathrm{~b}$ ). When mosquitoes were fed upon the viremic mice, the same outcome was observed (Fig. $2 \mathrm{~g}, \mathrm{~h}$ ). These results indicate that the four substitutions that directly reverted prior to the introduction of ZIKV into the Americas were major components of the fitness differences among African, Asian, and American virus strains. At the same time, similar competition assays were performed in human fibroblasts and keratinocytes. The ZIKV strains containing these four reverted amino acids always won the competition in these human primary cells (Supplementary Fig. 9).

To assess the roles of the individual amino acid substitutions in the overall fitness differences among ZIKV strains, we tested them individually using the same experimental systems. Placed individually into the African strain, all four initial mutations reduced overall fitness for infection, dissemination (two mutants showed no fitness effect in this compartment), and transmission by mosquitoes, although only C-A106T and NS1-V188A results were significant (Fig. $3 \mathrm{a}-\mathrm{c}$ ). The same trends were observed in mosquito bodies, legs, and saliva (Fig. $3 \mathrm{a}-\mathrm{c}$ ). When the reversions were placed into the Asian strain, only the NS1-A188V mutant produced a significant increase in ZIKV infection in the mosquito bodies, legs, and saliva, indicating this amino acid may have a dominant role during the competition (Fig. 3d-f). As with the combination of mutations, the lack of detection of one competitor in saliva samples indicates a major fitness advantage for vector transmission. To confirm fitness effects in models for the vertebrate amplification host, the Dakar NS1-V188A and FSS NS1-A188V mutants were mixed with corresponding $w t$ strains 
a

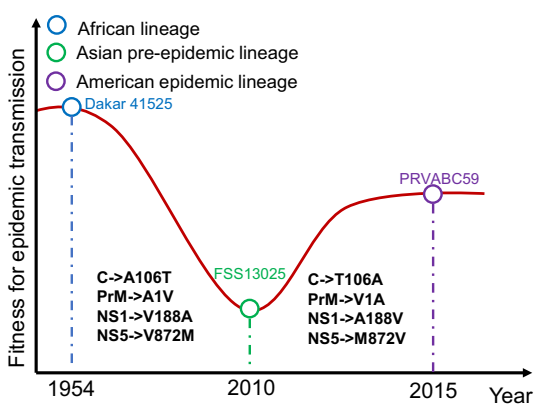

C

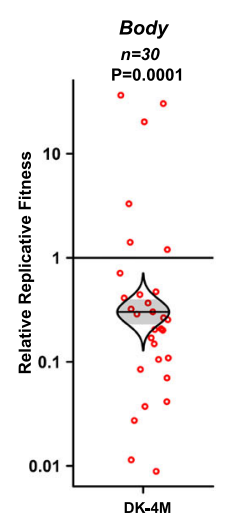

e

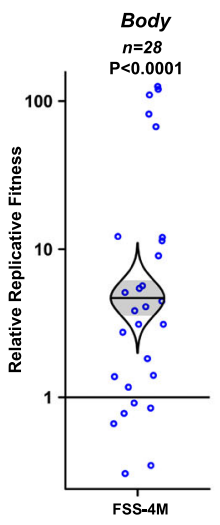

g

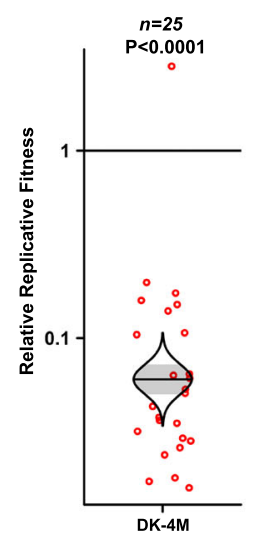

b
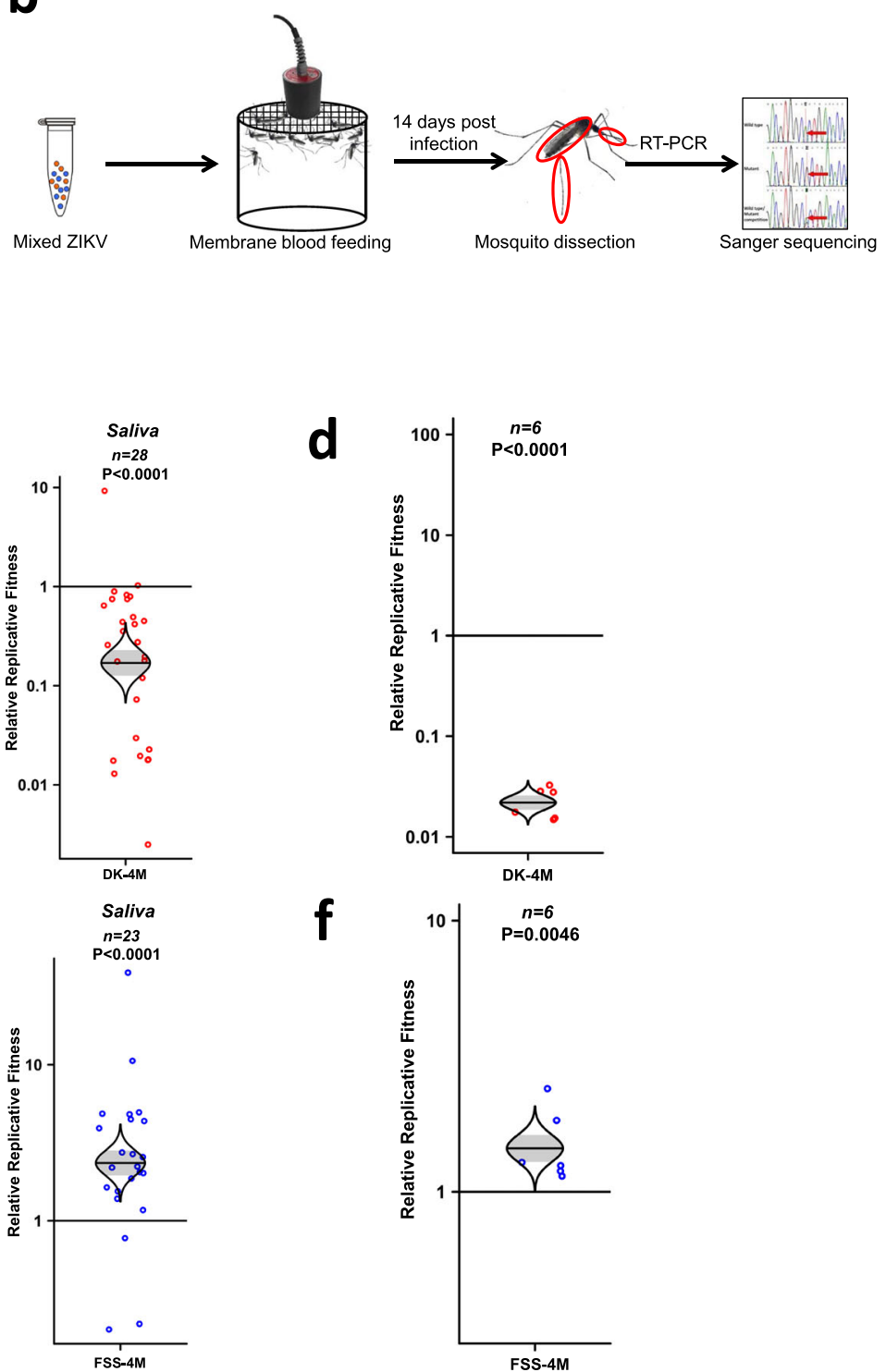

DK (African lineage)

FSS (Pre-epidemic Asian lineage) f
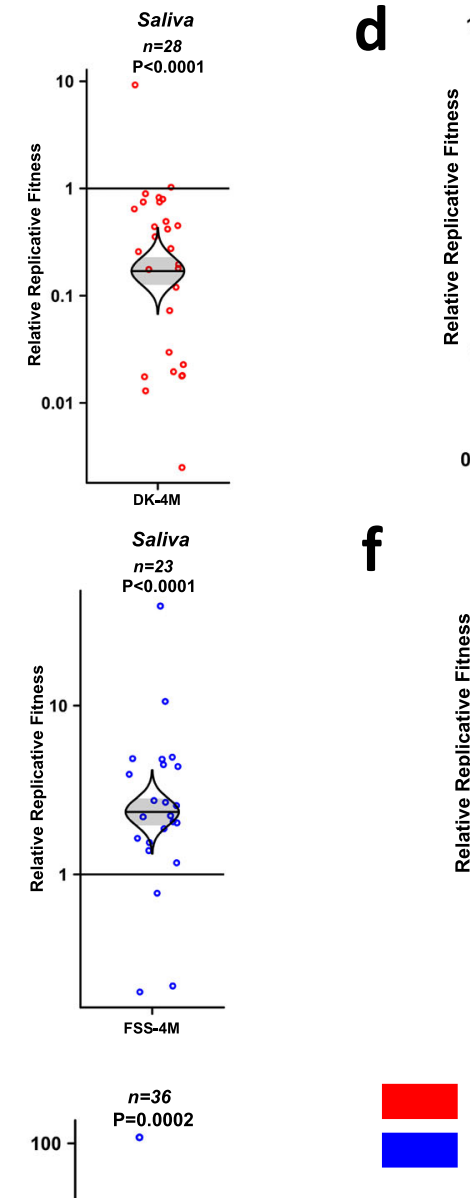

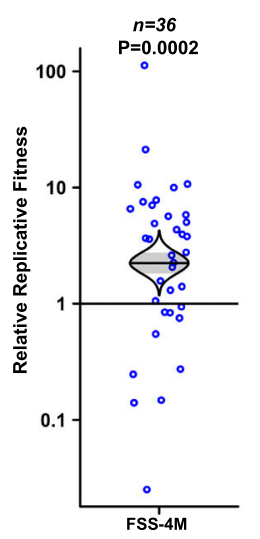

and inoculated into A129 mice. The ZIKVs containing NS1A226V exhibited a fitness advantage in both mice (Fig. 3g, h, Supplementary Fig. 10) and mosquitoes (Fig. 3i, j). The slight asymmetry in the fitness effects between some of the initial 4 mutations in the African strain and the reversions in the Cambodian pre-epidemic strain could reflect epistatic interactions that differ between the two virus strains. 
Fig. 2 Four amino acids determine much of the fitness difference between different ZIKV strains in both mosquitoes and A129 mice. a The hypothetical diagram of fitness changes following the spread of ZIKV from Africa to Asia and the Americas, including strains used for fitness assays. $\mathbf{b}$ Schematic representation of competition fitness assay in mosquito bodies, legs, and saliva. Figure adapted from Fig. 1a in Ref. ${ }^{14}$. c, e Fitness comparison between African and Asian lineage, and the four amino-acid substitution mutants (DK-4M and FSS-4M) in mosquito bodies, legs, and saliva. The mosquitoes acquired the ZIKV mixture through membrane blood-feeding and were assayed at 14 days post-infection. The body (carcass), legs, and saliva were collected from each individual mosquito and subjected to RT-PCR amplification and Sanger sequencing to determine relative fitness values of $4 \mathrm{M}$ over wt; each point represents a single mosquito or mouse sample. d, f Competition between DK-WT and DK-4M (d), FSS-WT, and FSS-4M (f) in mouse blood collected 3 days after infection. $\mathbf{g}, \mathbf{h}$ Fitness of DK-4M ( $\mathbf{g})$ and FSS-4M (h) in mosquitoes after oral infection from viremic mice. The mosquitoes acquired the virus through biting a viremic mouse 3 days after infection and were tested after 14 days of extrinsic incubation. $\mathbf{c}-\mathbf{h}$, The distribution of the modeladjusted means is illustrated by catseye plots with shaded \pm standard error (SE) overlaid by scatterplots of individual measures, which are shown on the log (base-10) scale such that comparisons are against a null value of 1. $P$ values are calculated for the group (strain) coefficient for each linear regression model. $n$ numbers represent the biologically independent samples and shown on the top of each figure. Source data are provided as a Source Data file. $\mathbf{c}, \mathbf{e}$, $\mathbf{g}$ The results were pooled from 3 independent repeats. $\mathbf{d}$, $\mathbf{f}$ The results were collected from a single experiment. $\mathbf{h}$ The results were pooled from 4 independent repeats.

than oral infection), these mosquitoes were exposed to A129 mice for transmission. Three days later during peak viremia, naive mosquitoes fed on the mice, and were assayed after 14 days of extrinsic incubation as in the above experiments. In salivary glands following incubation after mosquito inoculation, the initial four mutations decreased the fitness of the African ZIKV strain (Fig. 4b), and the four reversions increased the fitness of the Asian strain (Fig. 4c) as observed after oral infection (Fig. 2c, e). These fitness effects continued during the infection of mouse blood and organs (Fig. 4d, e, Supplementary Fig. 11) and also in mosquitoes fed on these mice (Fig. $4 \mathrm{f}, \mathrm{g}$ ). Overall, these results demonstrate that the four initial mutations reduced the fitness of the African ZIKV strain through a complete transmission cycle, while the four reversions partially restored fitness.

\section{Discussion}

Summary of ZIKV evolution in Asia. In summary, we identified three ZIKV amino acids (C-106, prM-1, and NS5-872) that we show for the first time affect transmission by increasing infection and transmission efficiency by $A$. aegypti and/or enhancing replication in models for human infection. We also demonstrate for the first time that, like those three residues, the NS1-A188V shown earlier to enhance vector infection ${ }^{14}$, also represents a direct reversion of an initial mutation that occurred soon after ZIKV reached Asia.

All South Pacific and American ZIKV strains include all four reversions that we studied (Supplementary Fig. 1, Supplementary Extended Data Table 1), consistent with their fitness advantage for urban transmission that we measured experimentally. However, a few recent Asian strains have all four mutations, which begs the question of why only limited outbreaks have been detected there. Probably not coincidentally, the only Asian strains to include all four of the reversion mutations are: (1) Chinese strains imported from the South Pacific or the Americas ${ }^{33}$, 2016 strains from Singapore sampled during the largest urban outbreak ever detected in Southeast Asia, and close relatives sampled in Thailand (Supplementary Data 1); strains from both locations apparently arose in Southeast Asia ${ }^{34-36}$. Although the Singapore outbreak was smaller than those reported in the Americas, ZIKV herd immunity in the region, resulting from long-term endemic circulation that is rarely detected ${ }^{37}$, combined with the highly efficient Singapore public health and vector control programs, probably limited the scope of the epidemic. Additional, urban transmission-adaptive mutations inferred from our data, such as E-473M $\mathrm{M}^{16}$, may also increase urban transmission competence but are not yet widespread in Asia. Finally, most Asian strains with three of the 4 revertant mutations were missing C-T106A, which had one of the strongest phenotypes we measured (Fig. 3a-c).
All of these findings suggest that all four reversions combined with E-473M (Supplementary Data 1) and limited herd immunity are needed for efficient epidemic ZIKV transmission. The fact that the first detection of these four reversions in 2012-2013 did not all occur immediately before the 2013 ZIKV introduction into the South Pacific and Americas is entirely consistent with emergence involving a combination of adaptive mutations that predispose virus strains to epidemic initiation, along with stochastic factors such as chance introductions into naive, epidemic transmission-permissive locations. Also, the lower fitness of the combination of the 4 reversions that we tested compared to the American strains (Fig. 2) indicates that additional mutations, not representing direct reversions, may also have been involved in emergence into the Americas. These conserved amino acid substitutions in distal tree branches and their possible phenotypes are listed in Supplementary Data 2. Because these additional adaptive mutations such as E-V473M, and probably others, are absent in many Asian ZIKV strains (Supplementary Data 1), epidemic potential there may be more limited than in the Americas where the naive population combined with the complete set of adaptive mutations led to massive outbreaks.

Possible explanations for direct reversions. Overall, our results support the hypothesis that ZIKV underwent fitness declines upon its introduction into Asia many decades ago, and/or during its initial circulation there when a lack of recognized outbreaks suggests limited urban transmission. An alternative hypothesis not relying on drift is that ZIKV host range changed upon its introduction into Asia, followed by another change (or reversion in host usage) just before introduction into the Americas; both of these hypothetical changes could have resulted in positive selection that involved the same four amino acids, resulting in reversion. The simplest form of this hypothesis would be that a sylvatic cycle involving different vectors and vertebrate hosts from those in Africa was the initial form of Asian transmission, and the urban cycle only developed there recently, facilitated by the four reversions. Although there is no evidence of enzootic ZIKV transmission in Asia, and the 1966 ZIKV isolation from $A$. aegypti mosquitoes in Malaysia suggests that human-amplified transmission has been ongoing in Asia for many decades, the detection of a sylvatic cycle is difficult in the absence of extensive genetic divergence from urban strains. Regardless of whether the four revertant mutations represent drift or host-adaptive selection, our experimental findings indicate that they probably had a major impact on urban transmission potential. Continued ZIKV surveillance in Asia is needed to determine if the genotype representing the combination of the four revertant mutations has persisted there, which could increase the risk of further outbreaks. 

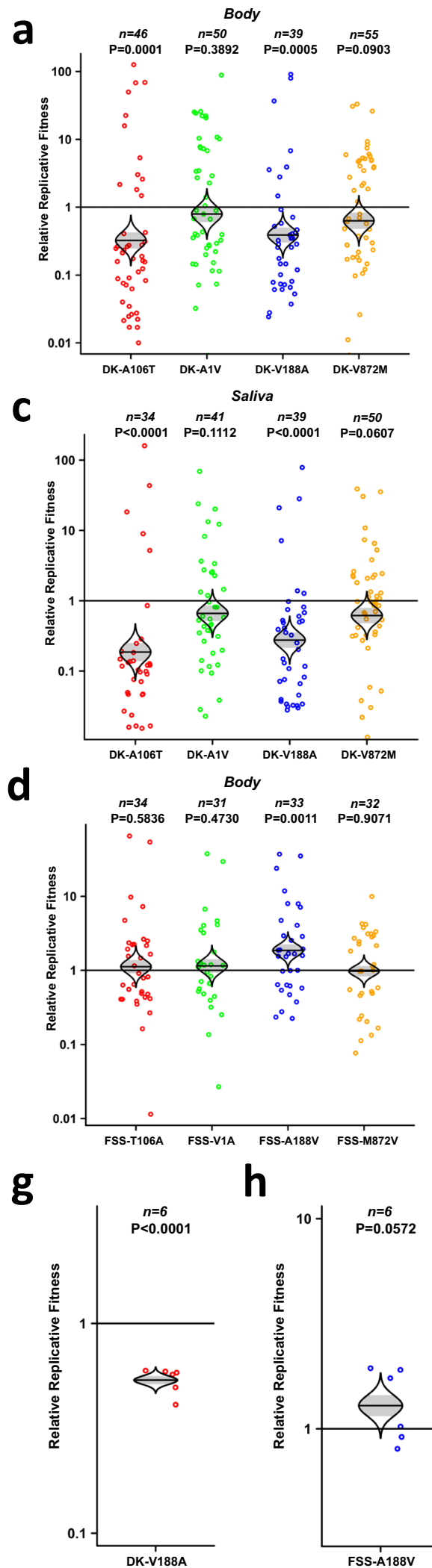

h

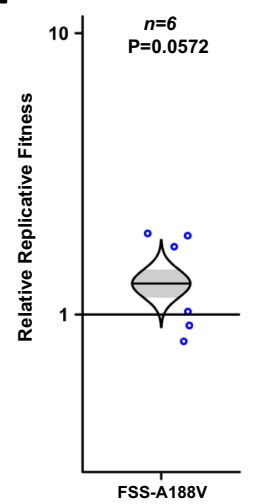

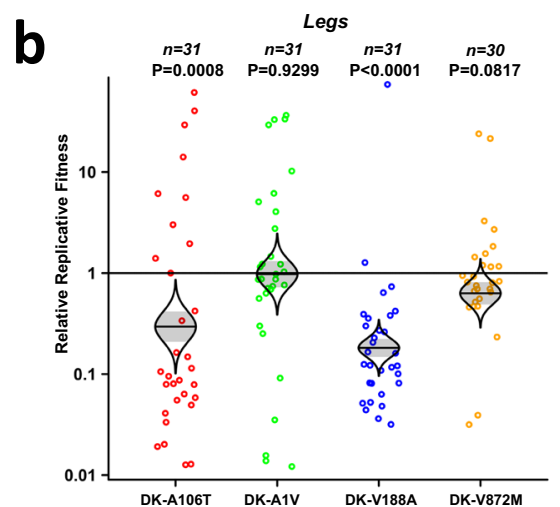

DK-A106T DK-A1V DK-V188A DK-V872M
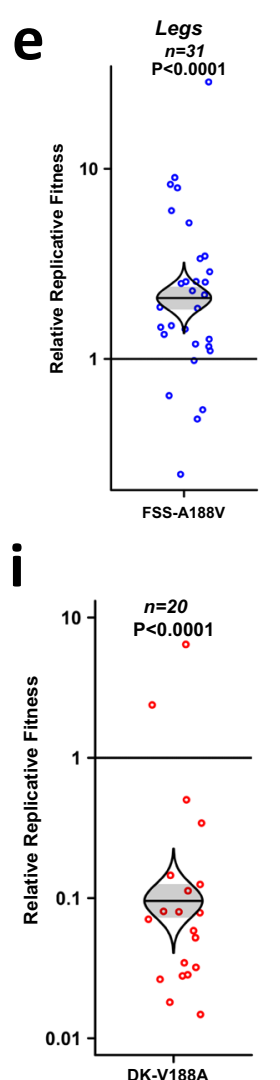

DK (African lineage)

FSS (Pre-epidemic Asian lineage)

Fig. 3 The fitness comparison of 4 individual mutant viruses against wild-type ZIKV strains in mosquitoes and A129 mice. a-c, The relative fitness of individual mutants in the Dakar strain, tested in mosquito bodies (a), legs (b), and saliva (c). d-f The fitness comparison of individual reversion mutants in the FSS13025 strain, tested in mosquito bodies (d), legs (e), and saliva (f). $\mathbf{g}$, $\mathbf{h}$ Each point represents a single mosquito sample. The fitness comparison of DK-V188A $(\mathbf{g})$ and FSS-A188V (h) versus wild-type strains in mouse blood. $\mathbf{i}, \mathbf{j}$, The fitness comparison of DK-V188A (i) and FSS-A188V (j) in mosquitoes after oral infection from viremic mice. $\mathbf{a}-\mathbf{j}$, The distribution of the model-adjusted means is illustrated by catseye plots with shaded \pm standard error (SE) overlaid by scatterplots of individual measures, which are shown on the log (base-10) scale such that comparisons are against a null value of 1. $P$ values are calculated for the group (strain) coefficient for each linear regression model. $n$ numbers represent biologically independent samples and shown on the top of each figure. Source data are provided as a Source Data file. $\mathbf{a}-\mathbf{f}, \mathbf{i}, \mathbf{j}$ The results were pooled from 3 independent repeats. $\mathbf{g}, \mathbf{h}$ The results were collected from a single experiment. 
Parallels with CHIKV. The hypothesis that the initial urban ZIKV cycle was inefficient due to founder effects and drift, is consistent with the same pattern of fitness loss that accompanied CHIKV introduction into Asia about a century ago (see details below). This fitness of this Asian lineage remains incompletely restored to this day ${ }^{38}$. Our results as well as the evolutionary history of ZIKV have remarkable parallels to those of CHIKV, which also evolved in Africa and spread many decades ago to Asia before its recent introduction into the Americas ${ }^{12}$. Previous studies indicate that the CHIKV arrived in Asia many decades ago with debilitating deletion mutations in its 3' untranslated genome region ( $3^{\prime} \mathrm{UTR}$ ), followed by only partial fitness restoration over many decades through point mutations and a duplication ${ }^{38}$. Another mutation in the E1 glycoprotein, also a probable founder effect (no phenotype has been established for this mutation), prevented for many decades CHIKV adaptation for efficient transmission by A. albopictus mosquitoes in Asia, their native territory ${ }^{39}$. Then, just before or upon its introduction into the Americas in 2013, a duplication in the 3'UTR improved CHIKV fitness ${ }^{40}$. However, unlike ZIKV, another lineage of CHIKV has undergone a series of adaptive mutations to enhance its ability to use A. albopictus for transmission, with no evidence of founder effects, and the relative fitness advantages of the CHIKV mutations far exceed those of the four ZIKV mutations that we studied; for example, individual A. albopictus-adaptive CHIKV mutations showed relative fitness values of 5-40, while even the combination of 4 ZIKV mutations we studied had a lower overall fitness effect than any of these individual CHIKV mutations (Supplementary Data 3). The similarities in the presumed roles of founder effects in the evolution and emergence potential of ZIKV and CHIKV suggest that drift has been understudied as a factor in the emergence of arboviral and other RNA viral diseases, and that the stochastic nature of founder effects may limit our ability to ultimately predict the emergence of new viral diseases.

Finally, the high fitness of the African ZIKV strains in all experimental systems we utilized raises important questions about the risk of outbreaks and severe disease on that continent. The greater transmissibility of these African strains, even compared to American strains ${ }^{21}$, raises the question of why outbreaks have never been detected in Africa aside from one in Angola caused by a strain imported from the Americas ${ }^{41}$. However, many African populations of $A$. aegypti have poor competence for ZIKV transmission ${ }^{42}$. Limited African surveillance and the widespread presence of other infections easily confused with Zika in the absence of laboratory diagnostics could be responsible. Herd immunity in Africa, which could also play a role in limiting outbreaks as well as the incidence of congenital Zika syndrome, has been reported to range from $1-52 \%{ }^{43}$. However, some studies have used methods that could be highly cross-reactive with other endemic flaviviruses. We also cannot rule out the possibility that our models for human infection are not suitable to determine ZIKV fitness for human amplification. Additional surveillance and work with more human cells and possibly nonhuman primate models are needed to further explore this possibility and to better understand the lack of Zika outbreaks in Africa caused by African lineage strains.

In addition to the fitness for transmission that we examined, the history of ZIKV's ability to cause GBS and CZS requires further study. It is possible that enhanced viremia just before spread to the Americas, suggested by our murine and human cells models, could at least partially explain the emergence of these severe disease outcomes if viremia magnitude is correlated with infection of the placenta. However, better surveillance in Africa is ultimately needed to determine if American or recent Asian strains are unique in this pathogenic potential.

\section{Methods}

Ethics statement. Mouse studies were performed in accordance with the $\mathrm{NIH}$ Guidance for the Care and Use of Laboratory Animals. The protocol (1708051) was approved by the University of Texas Medical Branch Institutional Animal Care and Use Committee under protocol 1708051. All mouse manipulations were performed under anesthesia with isoflurane.

Mice, mosquitoes, cells, and viruses. A129 mice, which are deficient in type I interferon receptors, were bred and maintained in animal biosafety level 2 (ABSL2) facilities at UTMB. Sex-matched mice, randomly selected and 6-to-8-week-old, were used. The Aedes aegypti Rockefeller strain and A. aegypti Dominican Republic strain (F6) were maintained in an incubator at $28^{\circ} \mathrm{C}$ and $80 \%$ humidity. Vero cells (CCL81) and C6/36 cells for virus rescue, proliferation, and titration were purchased from the American Type Culture Collection (Bethesda, MD, USA) and maintained in Dulbecco's modified Eagle's medium (Gibco, Waltham, MA, USA) with $10 \%$ heat-inactivated fetal bovine serum (Atlanta Biologicals, Flowery Branch, GA, USA), and supplemented with $1 \%$ Tryptose phosphate broth (Gibco, Waltham, MA, USA) for C6/36 cells. The human primary dermal fibroblast cells and human epidermal keratinocyte cells were purchased from Lonza (Walkersville MD, USA) and were maintained in FGM-2 BulletKit (Lonza) and KGM-Gold BulletKit (Lonza) media, respectively. All cells were verified and tested negative for mycoplasma. The parental African lineage ZIKV Dakar 41662 strain (KU955592), and 41671 stain (KU955595), and Asian lineage ZIKV Dominican Republic R114916 (KX766028), and Honduras HN-ME 59 stains were obtained from the World Reference Center for Emerging Viruses and Arboviruses at UTMB. The ZIKV DK-WT (Dakar wild-type 41525 strain, KU955591), DK-A106T (capsid 106th amino-acid), DK-A1V (pre-membrane 1st amino-acid), DK-V188A (nonstructural protein 1 188th amino-acid), DK-V872M (nonstructural protein 5 872nd amino-acid) were constructed using standard site-directed mutagenesis of cDNA clones and rescued on Vero cells using electroporation of transcribed RNA The FSS-WT (FSS13025 wild-type strain, KU955593), FSS-T106A, FSS-V1A, FSSA188V and FSS-M872V, as well as reversion mutants placed in the ZIKV Dakar strain, were constructed in the same manner, along with the DK-4M and FSS-4M that combined all four mutations on the matched backbone.

Competition assay. To better understand the relative fitness of different ZIKV strains and mutants, we conducted competition assays ${ }^{28,44}$ between two virus strains or a wild-type and mutant strain in various models. Initial 1:1 virus:mutant mixtures were made based on PFU titers determined in Vero cells, and virus ratios used to calculate fitness were determined by Sanger sequencing of RT-PCR amplicons. The PFU:genome ratio was consistent among all of our ZIKV strains and constructs, as estimated from the inocula and bloodmeals by comparing Vero cell PFU to genome copies as estimated from real-time RT-qPCR standard curves. To confirm that ratios were also similar in mosquito experimental systems, where RNA:infectious virus ratios could differ based on different levels of infectivity compared to Vero cells, we also determined infectious titers of the wt and mutants on C6/36 mosquito cells, and compared them with the Vero-PFU ratios.

Mosquitoes. The Aedes aegypti Rockefeller strain and A. aegypti Dominican Republic strain (F6) were used for the following studies. Triplicate mixtures of viruses were diluted to $6 \log _{10} \mathrm{PFU} / \mathrm{ml}$ mixed at a $1: 1$ ratio based on Vero PFU titers. Virus mixtures were subsequently mixed at a ratio of 1:1 with PBS-washed sheep blood cells (Hemostat laboratories, Galveston, TX, USA). Aliquots of blood meals were collected to verify the initial virus proportions in the inoculum. Following a 14-day extrinsic incubation, saliva expectorated into capillary tubes, legs representing virus disseminated into the hemocoel, or remaining mosquito bodies were collected separately in a $2 \mathrm{ml}$ Eppendorf Safe Lock tube with $250 \mu \mathrm{l}$ DMEM ( $2 \%$ FBS, $1 \%$ Sodium Pyruvate) with $25 \mathrm{mg} / \mathrm{ml}$ Amphotericin B and a stainless steel bead (Qiagen, Hilden, Germany). Samples were stored at $-80^{\circ} \mathrm{C}$.

A129 mice. Sex-matched A129 mice (6-8 weeks old) were used for ZIKV com petition assays.

The A129 mouse is defective for the interferon type I receptor and susceptible to ZIKV infection in vivo with high titered viremia. Mixtures of ZIKV strains (total $1 \times 10^{4} \mathrm{PFU} /$ mouse) were inoculated intradermally to simulate a mosquito bite. Titers of viruses before mixing were plaque assayed to verify proper concentrations Also, an aliquot of the inoculum was reserved for estimating the initial ratio of viruses. Mice were bled retro-orbitally on day 3 post-infection representing the peak. Serum was collected from blood via centrifugation at $2000 \mathrm{~g}$ for $5 \mathrm{~min}$ and stored at $-80^{\circ} \mathrm{C}$ until analysis. Eight days post-infection, all infected mice were killed and necropsied and major organs collected in a $2 \mathrm{ml}$ Eppendorf Safe Lock tube with $500 \mu \mathrm{l}$ DMEM ( $2 \%$ FBS, $1 \%$ Sodium Pyruvate) and a stainless-steel bead. Samples were stored at $-80^{\circ} \mathrm{C}$.

Human primary cells. The human fibroblasts and keratinocytes were purchased and qualified by ATCC. Cells were seeded into 12-well plates 1 day before infection to allow them to reach $90 \%$ confluence. Mixtures of ZIKVs (1 PFU/cell MOI) were added into the cells and incubated at $37^{\circ} \mathrm{C}$ for $2 \mathrm{~h}$. Titers of viruses before mixing were plaque assayed to verify proper concentrations. Also, an aliquot of the inoculum was reserved for estimating the initial ratio of the viruses. After 
a
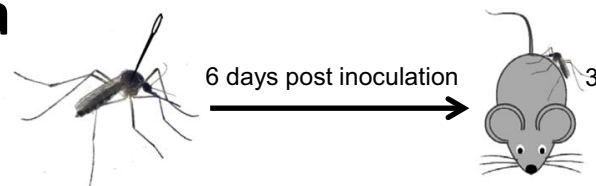

Infected mosquitoes

10 pfu of ZIKV WT and mutant viruses were intrathoracic injected into mosquitoes

b

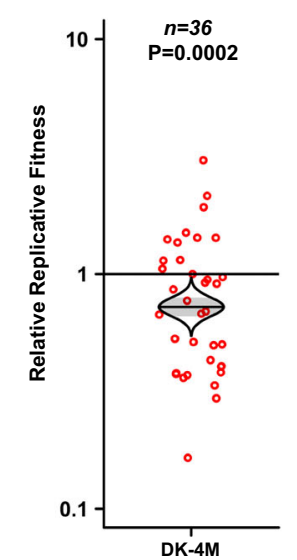

d

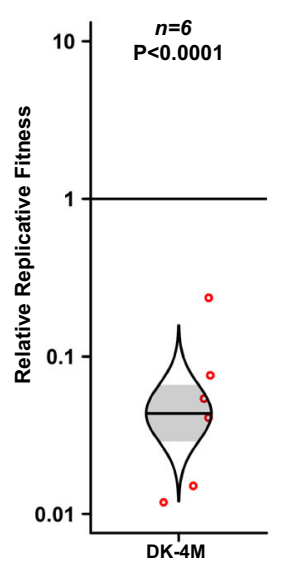

f

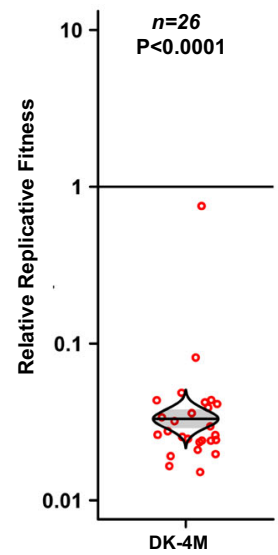
transmitted ZIKV to a A129 mouse through biting g

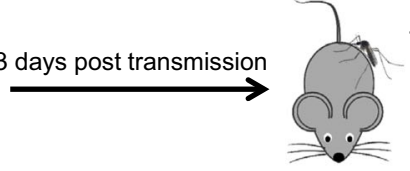

Mosquitoes acquire ZIKV 3 days post mouse infection
14 days post mosquito acquiring ZIKV

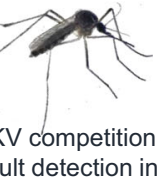
result detection in
mosquitoes
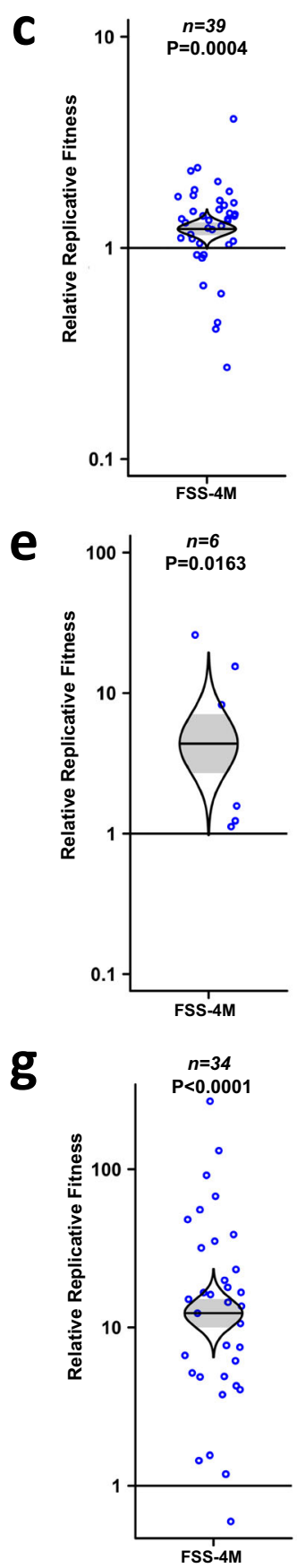

incubation, the cells were washed 3 times with $1 \mathrm{ml}$ of PBS. The infected cells were cultured, and the supernatant was collected from days 1 to 5 . Samples were stored at $-80^{\circ} \mathrm{C}$ for further analysis.

Sample preparation and Nucleic acid extraction. Aliquots of mosquito lysates, mouse serum, mouse organ lysates, and human primary cell supernatant samples were prepared in RNeasy Mini Kit (Qiagen) lysis buffer RLT ( $400 \mu \mathrm{l})$, and nucleic acids were subsequently extracted according to the manufacturer's protocol. After extraction, a portion of the RNA was immediately applied to a one-step RT-PCR assay and the remaining material was archived at $-80^{\circ} \mathrm{C}$.

Quantitative real-time RT-PCR assays. Prior to Sanger sequencing analysis, virus-positive samples were identified by quantitative real-time RT-PCR, which was performed using a QuantiTect Probe RT-PCR Kit (Qiagen) on the LightCycler 480 system (Roche, Rotkreuz, Switzerland) following the manufacturer's protocol. The primers and probes are listed in Supplementary Data 4. The absolute 
Fig. 4 Fitness comparison of DK-4M and FSS-4M against wild-type ZIKV strains during a mosquito-host-mosquito transmission cycle. a Schematic representation of the study design. 10 pfu of ZIKV WT and mutant viruses were intrathoracically injected into mosquitoes. After 6 days of extrinsic incubation, the infected mosquitoes fed on a A129 mice to transmit virus. Naive mosquitoes were then employed to bite the infected A129 mice 3 days post-mouse infection. After an additional 14 days, the mosquitoes were harvested. All the mosquitoes and mice samples collected during the transmission cycle were analyzed by RT-PCR and Sanger sequencing. Each point represents a single mosquito or mouse sample. Figure adapted from Fig. 1a in Ref. ${ }^{14}$. $\mathbf{b}$, c Fitness comparison of DK-4M (b) and FSS-4M (c) against wild-type viruses in mosquito salivary glands 6 days post-intrathoracic injection. $\mathbf{d}$, e Fitness of DK-4M (d) and FSS-4M (e) versus wild-type viruses in mouse blood. $\mathbf{f}, \mathbf{g}$ The fitness comparison of DK-4M (f) and FSS-4M (g) versus wild-type viruses in mosquitoes after oral infection from viremic mice. $\mathbf{b}-\mathbf{g}$ The distribution of the model-adjusted means is illustrated by catseye plots with shaded \pm standard error (SE) overlaid by scatterplots of individual measures, which are shown on the log (base-10) scale such that comparisons are against a null value of $1 . P$ values are calculated for the group (strain) coefficient for each linear regression model. $n$ numbers represent the biologically independent samples and shown on the top of each figure. Source data are provided as a Source Data file. $\mathbf{b}, \mathbf{c}$ The results were pooled from 4 independent repeats. $\mathbf{d}$, e The results were collected from a single experiment. $\mathbf{f}, \mathbf{g}$ The results were pooled from 3 independent repeats.

quantification of ZIKV RNA was determined using a standard curve with in vitrotranscribed, full-length ZIKV RNAs.

Reverse transcriptase PCR. A total of $400-500$ bp of RT-PCR product were synthesized and amplified from extracted RNA using a SuperScript ${ }^{\text {thx }}$ III One-Step RT-PCR kit (Invitrogen, Carlsbad, CA, USA). $20 \mu \mathrm{l}$ reactions were assembled in PCR 8-tube strips through the addition of $10 \mu \mathrm{l} 2 \mathrm{X}$ reaction mix, $0.4 \mu \mathrm{l}$ SuperScript $^{\text {tw }}$ III RT/Platinum ${ }^{\text {mox }}$ Taq Mix, $0.8 \mu \mathrm{l} 10 \mu \mathrm{M}$ specific forward primer, $0.8 \mu \mathrm{l}$ $10 \mu \mathrm{M}$ specific reverse primer (see Supplementary Data 4 ), $4 \mu$ lof extracted RNA and $6 \mu$ Rnase-free water. RT was completed using the following protocol: (1) $55^{\circ} \mathrm{C}, 30 \mathrm{~min} ; 94^{\circ} \mathrm{C}, 2 \mathrm{~min} ;(2) 94^{\circ} \mathrm{C}, 15 \mathrm{~s} ; 60^{\circ} \mathrm{C}, 30 \mathrm{~s} ; 68^{\circ} \mathrm{C}, 1 \mathrm{~min} ; 40$ cycles; (3) $68^{\circ} \mathrm{C}, 5 \mathrm{~min}$; (4) indefinite hold at $4^{\circ} \mathrm{C}$. A total of $2 \mu \mathrm{l}$ of generated PCR products were loaded to $1 \%$ DNA agarose gel to verify the size. The left samples were purified by a QIAquick PCR Purification kit (Qiagen) according to the manufacturer's protocol. The concentration of RT-PCR products $>10 \mathrm{ng} / \mu \mathrm{l}$ were qualified following Sanger sequencing.

Sanger sequencing and electropherogram peak height analysis. Sequences of the purified RT-PCR products (concentration $>10 \mathrm{ng} / \mu \mathrm{l}$ ) were generated using a BigDye Terminator v3.1 cycle sequencing kit (Applied Biosystems, Austin, TX, USA). The products of the sequencing reactions were then purified using a 96-well plate format (EdgeBio, San Jose, CA, USA), and then analyzed on a 3500 Genetic Analyzer (Applied Biosystems). The peak electropherogram height representing each mutation site and proportion of each competitor was analyzed using the QSVanalyser program (version 20121206) ${ }^{45}$.

Intrathoracic inoculation of mosquitoes. For the intrathoracic microinjection of ZIKV into mosquitoes, female mosquitoes were anaesthetized on a cold tray $\left(0^{\circ} \mathrm{C}\right)$. Then, a defined titer ( $10 \mathrm{pfu}$ in $200 \mathrm{nl}$ total inoculum volume) of mixed ZIKV strains were inoculation into the mosquito thorax with a Nanoject III (Drummond, Pennsylvania, USA). Six days later, the infected mosquitoes were allowed to feed on A129 mice to transmit ZIKV 46 .

Membrane blood-feeding. Seven-day-old female mosquitoes were placed into mesh-covered cartons and provided cotton balls containing $10 \%$ sucrose. Complement-inactivated sheep blood was mixed with different ZIKV combinations for mosquito feeding via a Hemotek system membrane feeder (5W1, Hemotek limited, Lancashire, UK). The total viral titers of the two competing ZIKVs in the blood meals were $1 \times 10^{6} \mathrm{PFU} / \mathrm{ml}$. Fully engorged mosquitoes were incubated for 14 days until harvest. At that time, live mosquitoes were killed by freezing and homogenized individually (TissueLyser II, Qiagen) for RNA isolation and real-time qPCR detection. The infected mosquito RNAs were RT-PCR amplified, followed by Sanger sequencing.

Mosquito feeding on infected mice. Ten female mosquitoes were placed into mesh-covered cartons for blood-feeding following sugar-starvation for $24 \mathrm{~h}$. ZIKVinfected A129 mice were anaesthetized with ketamine and placed on the top of the cartons for $20 \mathrm{~min}$ of feeding in the dark. Then, fully engorged mosquitoes were transferred to new containers and incubated for 14 days prior to RT-PCR, followed by Sanger sequencing of amplicons.

Plaque assay. The plaque assay was performed on Vero cells. In brief, Vero cells were seeded into 12 -well plates $12-16 \mathrm{~h}$ prior to the plaque assay. A total of $25 \mu \mathrm{l}$ of each sample were used for 10 -fold serial dilutions. For each dilution, $200 \mu \mathrm{l}$ were added to 12 -well plates with $90 \%$ confluent Vero cells. The cells were incubated at $37^{\circ} \mathrm{C}$ with $5 \% \mathrm{CO}_{2}$ for $1 \mathrm{~h}$ with gentle shaking every $15 \mathrm{~min}$. After that, $1 \mathrm{ml}$ of overlay (DMEM, $2 \%$ FBS, containing $0.8 \%$ methyl cellulose with $1 \%$ antibiotics) was added onto each well. The plates were cultured at $37{ }^{\circ} \mathrm{C}$ with $5 \% \mathrm{CO}_{2}$ for 4 days until clear plaques formed. The plates were fixed in $4 \%$ formaldehyde solution for $2 \mathrm{~h}$ and stained with $1 \%$ crystal violet ${ }^{47}$.
Focus-forming assay (FFA). The FFA was performed on C6/36 mosquito cells. In brief, C6/36 cells were seeded into 12 -well plates $12-16 \mathrm{~h}$ prior to the assay. A $25 \mu \mathrm{l}$ volume of each sample was used for 10 -fold serial dilutions. For each dilution, 200 $\mu \mathrm{l}$ were added to 12 -well plates with $90 \%$ confluent cells. The cells were incubated at $30^{\circ} \mathrm{C}$ with $5 \% \mathrm{CO}_{2}$ for $1 \mathrm{~h}$ with gentle shaking every $15 \mathrm{~min}$. After a 5 -day incubation, plates were fixed with methanol/acetone (1:1), washed by PBS, and blocked with $2 \% \mathrm{FBS} / \mathrm{PBS}$ before overnight incubation with 1:2000 diluted mouse anti-ZIKV antibody (4G2). Plates were washed and incubated with 1:2000 diluted goat anti-mouse secondary antibody conjugated to horseradish peroxidase (HRP) (KPL, Gaithersburg, MD, USA), then washed and developed with aminoethyl carbazole solution (Enzo Diagnostics, Farmingdale, NY, USA) prepared according to the manufacturer's protocol for detection of infection foci ${ }^{21}$.

Construction of ZIKV mutant infectious clones. The ZIKV FSS13025 full-length cDNA infectious clone pFLZIKV-FSS ${ }^{47}$ (referred as FSS-WT in text) was used as the backbone for engineering FSS-T106A, FSS-V1A, FSS-A188V, FSS-M872V, and FSS-4M mutants. The ZIKV Dakar 41525 full-length cDNA infectious clone pFLZIKV-DK (referred as DK-WT in text) was used as the backbone for engineering DK-A106T, DK-A1V, DK-V188A, DK-V872M, and DK-4M mutants. Standard overlapping PCR was performed to amplify the cDNA fragment between unique restriction enzyme sites that contained the corresponding mutations. Afterward, the fragments were cloned into pFLZIKV-FSS or pFLZIKV-DK plasmids and propagated in E. coli strain Top 10 (ThermoFisher Scientific, Framingham, MA, USA). All restriction enzymes were purchased from New England BioLabs (Massachusetts, USA). The plasmids were validated by restriction enzyme digestion and Sanger DNA sequencing. All primers were synthesized from Integrated DNA Technologies and are available upon request.

RNA transcription, electroporation, and virus recovery. The full-length cDNA clone plasmids of all ZIKVs were linearized with restriction enzyme ClaI prior to RNA transcription. The linearized DNAs were purified by phenol-chloroform extraction and ethanol precipitation. RNA transcripts of ZIKVs were synthesized using the T7 mMessage mMachine kit (Ambion, California, USA) in vitro. The quantity and quality of RNAs were verified by Spectrophotometry (DS-11, DeNovix, Delaware, USA) and Agarose gel electrophoresis. For RNA transfection, $10 \mu \mathrm{g}$ of transcribed RNA were electroporated into $8 \times 10^{6}$ Vero cells using the Gene Pulser Xcell ${ }^{\text {is }}$ Electroporation Systems (Bio-rad, California, USA), in $4 \mathrm{~mm}$ cuvettes with $0.8 \mathrm{ml}$ of Ingenio Electroporation Solution (Mirus, Madison, USA). The cells were pulsed three times with $3 \mathrm{~s}$ intervals at $0.45 \mathrm{kV}$ and $25 \mu \mathrm{F}^{41}$. After 10 min recovery at room temperature, the cells were transferred into T-175 flask with normal culture medium and incubated at $37^{\circ} \mathrm{C}$ with $5 \% \mathrm{CO}_{2}$. The viral culture supernatant was harvested when obvious cytopathic effects (CPE) of the transfected Vero cells were observed, and the titers of all ZIKVs were measured on Vero cells using plaque assays ${ }^{46}$.

Statistics. Animals were randomly allocated to different groups. Mosquitoes that died before measurement were excluded from the analysis. The investigators were not blinded to the allocation during the experiments or to the outcome assessment. No statistical methods were used to predetermine the sample size. Descriptive statistics are provided in the figure legends. Linear regression analysis was used to assess the correlation between WT/Mutant ratios by Sanger sequencing and WT/ Mutant ratios by PFU. Analysis was performed in Prism version 7.03 (GraphPad, San Diego, 440 CA).

For virus competition experiments in mosquitoes, A129 mice and human primary cells, relative replicative fitness values for viruses in each sample were analyzed according to $\mathrm{w}=(\mathrm{f} 0 / \mathrm{i} 0)$, where $\mathrm{i} 0$ is the initial ratio of one competitor and fo is the final ratio after competition. Sanger sequencing (initial timepoint T0) counts for each virus strain being compared (for example, wild-type versus mutant strains) were based upon average counts over several replicate samples of inocula or bloodmeals per experiment (effectively averaging the replicates), and postinfection (timepoint T1) counts were taken from samples of individual subjects, 
such that for each strain the ratio between counts at T0 and T1 (T1/T0) reflects a measure of a sample from a single mosquito, mouse or cell culture well in a specific experiment for that strain. Typically, multiple experiments were performed, so that f0/i0 was clustered by experiment. To model f0/i0, the ratio T0/T1 was found separately for each subject in each strain group, log (base-10) transformed to an improved approximation of normality, and modeled by analysis of variance with relation to group, adjusting by experiment to control for clustering within the experiment. Specifically, the model was of the form Log10_CountT1overCountT0 $\sim$ Experiment + Group. Fitness ratios between the two groups [the model's estimate of $\mathrm{w}=(\mathrm{f} 0 / \mathrm{i} 0)]$ were assessed per the coefficient of the model's Group term, which was transformed to the original scale as $10^{\wedge}$ coefficient. This modeling approach compensates for any correlation due to clustering within experiment similarly to that of corresponding mixed effect models, and is effective since the number of experiments was small. Statistical analyses were performed using $\mathrm{R}$ statistical software (R Core Team, 2019, version 3.6.1). In all statistical tests, twosided alpha $=.05$. Catseye plots ${ }^{48}$, which illustrate the normal distribution of the model-adjusted means, were produced using the "catseyes" package 49 .

Reporting Summary. Further information on research design is available in the Nature Research Reporting Summary linked to this article.

\section{Data availability}

Source data for generating the main figures and supplementary figures are provided with this paper. Any other information is available upon request. Source data are provided with this paper.

Received: 17 November 2020; Accepted: 15 December 2020; Published online: 26 January 2021

\section{References}

1. Dick, G. W., Kitchen, S. F. \& Haddow, A. J. Zika virus. I. Isolations and serological specificity. Trans. R. Soc. Trop. Med. Hyg. 46, 509-520 (1952)

2. Grard, G. et al. Zika virus in Gabon (Central Africa)-2007: a new threat from Aedes albopictus? PLoS Negl. Trop. Dis. 8, e2681 (2014).

3. Duffy, M. R. et al. Zika virus outbreak on Yap Island, Federated States of Micronesia. N. Engl. J. Med 360, 2536-2543 (2009).

4. Cao-Lormeau, V. M. et al. Zika virus, French polynesia, South pacific, 2013. Emerg. Infect. Dis. 20, 1085-1086 (2014).

5. Oehler, E. et al. Zika virus infection complicated by Guillain-Barre syndrome-case report, French Polynesia, December 2013. Euro. Surveill. 19, 20720 (2014)

6. Campos, G. S., Bandeira, A. C. \& Sardi, S. I. Zika Virus Outbreak, Bahia, Brazil. Emerg. Infect. Dis. 21, 1885-1886 (2015).

7. Brasil, P. et al. Zika Virus Infection in Pregnant Women in Rio de Janeiro. $N$. Engl. J. Med. 375, 2321-2334 (2016).

8. Aliota, M. T. et al. Zika in the Americas, year 2: What have we learned? What gaps remain? A Rep. Glob. Virus Netw. Antivir. Res 144, 223-246 (2017).

9. Haddow, A. D. et al. Genetic characterization of Zika virus strains: geographic expansion of the Asian lineage. PLoS Negl. Trop. Dis. 6, e1477 (2012).

10. Moudy, R. M., Meola, M. A., Morin, L. L., Ebel, G. D. \& Kramer, L. D. A newly emergent genotype of West Nile virus is transmitted earlier and more efficiently by Culex mosquitoes. Am. J. Trop. Med. Hyg. 77, 365-370 (2007).

11. Brault, A. C. et al. Venezuelan equine encephalitis emergence: Enhanced vector infection from a single amino acid substitution in the envelope glycoprotein. Proc. Natl Acad. Sci. USA 101, 11344-11349 (2004).

12. Weaver, S. C., Charlier, C., Vasilakis, N. \& Lecuit, M. Zika, Chikungunya, and other emerging vector-borne viral diseases. Annu. Rev. Med. 69, 395-408 (2018).

13. Domingo, E. \& Holland, J. J. in Evolutionary Biology of Viruses (ed S. S. Morse) 161-184 (Raven Press, 1994).

14. Liu, Y. et al. Evolutionary enhancement of Zika virus infectivity in Aedes aegypti mosquitoes. Nature 545, 482-486 (2017).

15. Azar, S. R., Diaz-Gonzalez, E. E., Danis-Lonzano, R., Fernandez-Salas, I. \& Weaver, S. C. Naturally infected Aedes aegypti collected during a Zika virus outbreak have viral titres consistent with transmission. Emerg. Microbes Infect. 8, 242-244 (2019).

16. Shan, C. et al. A Zika virus envelope mutation preceding the 2015 epidemic enhances virulence and fitness for transmission. Proc. Natl Acad. Sci. USA 117, 20190-20197 (2020).

17. Yuan, L. et al. A single mutation in the prM protein of Zika virus contributes to fetal microcephaly. Science 358, 933-936 (2017).

18. Pettersson, J. H. et al. How Did Zika Virus Emerge in the Pacific Islands and Latin America? MBio. 7, (2016).
19. Mier, Y. T.-R. L., Tatem, A. J. \& Johansson, M. A. Mosquitoes on a plane: Disinsection will not stop the spread of vector-borne pathogens, a simulation study. PLoS Negl. Trop. Dis. 11, e0005683 (2017).

20. Duarte, E. A. et al. Subclonal components of consensus fitness in an RNA virus clone. J. Virol. 68, 4295-4301 (1994).

21. Roundy, C. M. et al. Variation in Aedes aegypti mosquito competence for Zika virus transmission. Emerg. Infect. Dis. 23, 625-632 (2017)

22. Weger-Lucarelli, J. et al. Vector Competence of American mosquitoes for three strains of Zika virus. PLoS Negl. Trop. Dis. 10, e0005101 (2016).

23. Smith, D. R. et al. African and Asian Zika virus isolates display phenotypic differences both in vitro and in vivo. Am. J. Trop. Med. Hyg. 98, 432-444 (2018).

24. Duggal, N. K. et al. Differential Neurovirulence of African and Asian genotype Zika virus isolates in outbred immunocompetent mice. Am. J. Trop. Med. Hyg. 97, 1410-1417 (2017).

25. Wiser, M. J. \& Lenski, R. E. A comparison of methods to measure fitness in Escherichia coli. PLoS ONE 10, e0126210 (2015).

26. Grubaugh, N. D. et al. Genetic drift during systemic Arbovirus infection of mosquito vectors leads to decreased relative fitness during host switching. Cell Host Microbe 19, 481-492 (2016).

27. Bergren, N. A. et al. "Submergence" of western equine encephalitis virus: evidence of positive selection argues against genetic drift and fitness reductions. PLoS Path 16, e1008102 (2019).

28. Plante, J. A. et al. Spike mutation D614G alters SARS-CoV-2 fitness and neutralization susceptibility. Nature https://doi.org/10.1038/s41586-020-2895-3 (2020).

29. Lazear, H. M. et al. A mouse model of Zika virus pathogenesis. Cell Host Microbe 19, 720-730 (2016)

30. Rossi, S. L. et al. Characterization of a novel murine model to study Zika virus. Am. J. Trop. Med. Hyg. 94, 1362-1369 (2016).

31. Hamel, R. et al. Biology of Zika virus infection in human skin cells. J. Virol. 89 , 8880-8896 (2015)

32. Kim, J. A., Seong, R. K., Son, S. W. \& Shin, O. S. Insights into ZIKV-mediated innate immune responses in human dermal fibroblasts and epidermal keratinocytes. J. Invest. Dermatol. 139, 391-399 (2019).

33. Zhang, Y. et al. Highly diversified Zika viruses imported to China, 2016 Protein Cell 7, 461-464 (2016).

34. Maurer-Stroh, S. et al. South-east Asian Zika virus strain linked to cluster of cases in Singapore, August 2016. Euro. Surveill. 21, https://doi.org/10.2807/ 1560-7917.ES.2016.21.38.30347 (2016)

35. Singapore Zika Study, G. Outbreak of Zika virus infection in Singapore: an epidemiological, entomological, virological, and clinical analysis. Lancet Infect. Dis. 17, 813-821 (2017).

36. Wongsurawat, T. et al. Case of microcephaly after congenital infection with Asian Lineage Zika Virus, Thailand. Emerg. Infect. Dis. 24, 1758-1761 (2018)

37. Ruchusatsawat, $K$. et al. Long-term circulation of Zika virus in Thailand: an observational study. Lancet Infect. Dis. 19, 439-446 (2019).

38. Chen, R., Wang, E., Tsetsarkin, K. A. \& Weaver, S. C. Chikungunya virus 3 untranslated region: adaptation to mosquitoes and a population bottleneck as major evolutionary forces. PLoS Pathog. 9, e1003591 (2013).

39. Tsetsarkin, K. A. et al. Chikungunya virus emergence is constrained in Asia by lineage-specific adaptive landscapes. Proc. Natl Acad. Sci. USA 108, 7872-7877 (2011).

40. Stapleford, K. A. et al. Whole-genome sequencing analysis from the Chikungunya Virus Caribbean outbreak reveals novel evolutionary genomic elements. PLoS Negl. Trop. Dis. 10, e0004402 (2016)

41. Hill, S. C. et al. Emergence of the Asian lineage of Zika virus in Angola: an outbreak investigation. Lancet Infect. Dis. 19, 1138-1147 (2019).

42. Aubry, F. et al. Enhanced Zika virus susceptibility of globally invasive Aedes aegypti populations. Science 370, 991-996 (2020).

43. Saiz, J. C. et al. Zika Virus: the Latest Newcomer. Front Microbiol. 7, 496 (2016)

44. Tsetsarkin, K. A. et al. Multi-peaked adaptive landscape for chikungunya virus evolution predicts continued fitness optimization in Aedes albopictus mosquitoes. Nat. Commun. 5, 1-14 (2014).

45. Carr, I. M. et al. Inferring relative proportions of DNA variants from sequencing electropherograms. Bioinformatics 25, 3244-3250 (2009).

46. Liu, J. et al. Flavivirus NS1 protein in infected host sera enhances vira acquisition by mosquitoes. Nat. Microbiol. 1, 1-11 (2016).

47. Shan, C. et al. An infectious cDNA clone of Zika virus to study viral virulence, mosquito transmission, and antiviral inhibitors. Cell Host Microbe 19, 891-900 (2016).

48. Cumming, G. (2014). The new statistics: why and how. Psychol. Sci. 27, 7-29 (2014).

49. Andersen, C. catseyes: Create Catseye Plots Illustrating the Normal Distribution of the Means. R package version 0.2.3. (2019).

\section{Acknowledgements}

S.C.W. was supported by NIH grants AI120942 and AI121452. P.-Y.S. was supported by NIH grants AI142759, AI127744, and AI136126, and awards from the Kleberg Foundation, 
John S. Dunn Foundation, Amon G. Carter Foundation, Gilson Longenbaugh Foundation, and Summerfield Robert Foundation. J. L. was supported by the McLaughlin Fellowship Fund. We thank Aaron Brault for helpful suggestions for the manuscript.

\section{Author contributions}

S.C.W., J.L., Y.L., C.S., and P.-Y.S. designed the experiments and/or wrote the manuscript; J.L. and Y.L. performed the majority of the experiments and analyzed the data; S.C., B.T.D.N., and P.-Y.S. developed the reverse genetic system for ZIKV strains; R.M. and N.V. provided the mosquito colonies; R.M. assisted in mosquito collection; S.L.H assisted in designing the competition assay; G.H.R. and S.R.A provide the A129 mice colonies; C.R.A. assisted with statistics analyses; K.P. provided ZIKV parental strains and contributed experimental suggestions. All authors reviewed, critiqued, and provided comments on the text.

\section{Competing interests}

The authors declare no competing interests.

\section{Additional information}

Supplementary information is available for this paper at https://doi.org/10.1038/s41467020-20747-3.

Correspondence and requests for materials should be addressed to P.-Y.S. or S.C.W.
Peer review information Nature Communications thanks the anonymous reviewers for their contribution to the peer review of this work.

Reprints and permission information is available at http://www.nature.com/reprints

Publisher's note Springer Nature remains neutral with regard to jurisdictional claims in published maps and institutional affiliations.

(c) Open Access This article is licensed under a Creative Commons Attribution 4.0 International License, which permits use, sharing, adaptation, distribution and reproduction in any medium or format, as long as you give appropriate credit to the original author(s) and the source, provide a link to the Creative Commons license, and indicate if changes were made. The images or other third party material in this article are included in the article's Creative Commons license, unless indicated otherwise in a credit line to the material. If material is not included in the article's Creative Commons license and your intended use is not permitted by statutory regulation or exceeds the permitted use, you will need to obtain permission directly from the copyright holder. To view a copy of this license, visit http://creativecommons.org/ licenses/by/4.0/.

(C) The Author(s) 2021 